\title{
INCIDÊNCIA DE LESÕES MUSCULOESQUELÉTICAS EM ATLETAS MASCULINOS DE HANDEBOL
}

\author{
INCIDENCE OF MUSCULOSKELETAL INJURIES IN MALE HANDBALL ATHLETES
}

\section{Danilo Harudy Kamonseki ${ }^{a^{*}}$, Carlos Luques Fonseca ${ }^{b^{* *}}$, Antonio Roberto Zamunér ${ }^{\mathrm{c}^{* * *}}$, Neemias Adão Souza ${ }^{\mathrm{d}^{* *}}$, Geiseane Gonçalves Aguiar ${ }^{\mathrm{e}^{* * *}}$, Beatriz Oliveira Peixoto ${ }^{\mathrm{f}^{*}}$}

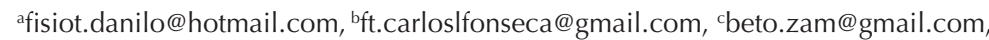

dneemyas.s.a@hotmail.com, egeiseane.agoncalves@gmail.com, ffisiosorocaba@unip.br

*Universidade Paulista - Sorocaba (SP), Brasil

**Faculdade Anhanguera - Sorocaba (SP), Brasil

***Universidade Federal de São Carlos - São Carlos (SP), Brasil

****Universidade Federal de São Paulo - Santos (SP), Brasil

Data de recebimento do artigo: 25/10/2014

Data de aceite do artigo: 08/04/2015

\section{RESUMO}

Introdução: O Handebol é um esporte rápido, explosivo e de contato, que exige açôes como saltar, bloquear, realizar sprints e arremessar, sendo considerado de alto risco para a ocorrência de lesôes. Objetivo: verificar a incidência de lesóes musculoesqueléticas que ocorreram durante uma temporada em atletas de handebol. Materiais e métodos: Foi realizado um estudo observacional prospectivo, referente à incidência das lesóes musculoesqueléticas em atletas do gênero masculino, com idade de 16 a 20 anos, durante a temporada de 2010. Resultados: A incidência total foi de 13,06 lesóes a cada 1000 horas de exercício, sendo que durante os jogos, foi de 40,81 lesôes, e nos treinos, 8,24. A região mais acometida foi o tornozelo $(41,66 \%)$, seguido pelo ombro $(25 \%)$, joelho $(12,5 \%)$, cabeça $(8,33 \%)$, mão $(4,16 \%)$, coxa $(4,16 \%)$ e cotovelo (4,16\%). Conclusáo: Este estudo observou que as lesóes predominantes no handebol acometem os membros inferiores, seguidos pelos superiores, sendo a maioria considerada leve. A quantidade de lesôes durante os treinos foi maior, mas quando consideradas por hora praticada, os jogos foram mais lesivos.

Palavras-chave: Epidemiologia; traumatismos em atletas; taxa de incidência.

\section{ABSTRACT}

Introduction: Handball is a fast-paced, explosive and contact sport, which requires actions such as jumping, blocking and throwing, considered a high-risk sport, for its occurrence of injuries. Objective: To verify the incidence of musculoskeletal injuries occurred in a season with handball players. Materials and methods: It was an observational prospective study, relative the occurrence of musculoskeletal injuries in male athletes, aged 16 to 20 years, during the 2010 season. Results: The total incidence was 13.06 injuries per 1000 hours of playing; during matches, 40.81 injuries, and during practices, 8.24 . The most affected region of the body was the ankle $(41,66 \%)$, followed by shoulder $(25 \%)$, knee $(12,5 \%)$, head $(8,33 \%)$, hand $(41,6 \%)$, thigh $(4,16 \%)$ and elbow (4,16\%). Conclusion: The predominant injuries in handball affects the lower limbs followed by superior ones, although most of injuries were considered mild. The amount of injuries during practices was higher, but when considered injuries per hour played, matches were more harmful.

Keywords: Epidemiology; athletic injuries; incidence rate. 


\section{Introdução}

Handebol é um esporte rápido e explosivo, que exige açóes como saltar, fintar, bloquear, arremessar e correr em diferentes direçôes e velocidades, e com frequência há contato físico entre os atletas, sendo considerado de alto risco para a ocorrência de lesóes ${ }^{1,2}$, podendo causar afastamentos da prática esportiva e elevados custos com tratamentos e reabilitação ${ }^{3}$.

Essas lesôes podem ser evitadas por meio de estratégias e programas preventivos, desenvolvidos com base em fatores de risco ${ }^{3}$. Esses fatores são característicos de cada esporte, assim, estudos epidemiológicos são necessários para compreender e determinar a gravidade, as caractísticas e os mecanismos que estão associados às lesões ${ }^{4}$.

Os estudos epidemiológicos sobre o handebol são poucos, e os resultados dos trabalhos existentes são de difícil comparação, pela variedade dos modelos de pesquisa, definição de lesão, período observado e nível de jogo $^{1}$. Em alguns estudos, as lesóes foram avaliadas por meio de questionários ou entrevistas retrospectivas ${ }^{5-8}$, análise de vídeos ${ }^{9}$, durante uma temporada ${ }^{10,11}$ ou em torneios internacionais ${ }^{1,9}$.

Nesse contexto, o objetivo deste estudo foi verificar a incidência e as características das lesôes em atletas masculinos de handebol que ocorreram na temporada de 2010.

\section{Casuística e métodos}

Foi realizado um estudo observacional prospectivo, referente à incidência das lesões musculoesqueléticas em atletas de uma equipe de handebol, com idade entre 16 e 20 anos, do gênero masculino, que apresentavam resultados competitivos expressivos em nível estadual. Foram excluídos os voluntários que faltaram aos treinos ou à competição por motivo de doenças sistêmicas ou outras condiçôes médicas.

Este estudo foi aprovado pelo Comitê de Ética e Pesquisa em Seres Humanos da Universidade Paulista - UNIP (Protocolo: 630/10) e os voluntários assinaram o Termo de Consentimento Livre e Esclarecido, após serem informados de todos os procedimentos do estudo.

Os dados foram coletados durante uma temporada de competiçóes, de fevereiro a novembro de 2010. A frequência de partidas foi de um jogo por semana e quatro treinos semanais com duas horas e meia de duração cada, incluindo os treinos físicos e táticos.

Foram consideradas e classificadas como lesóes musculoesqueléticas as que acometiam o aparelho locomotor dos atletas, levando ao afastamento total ou parcial do treinamento ou competiçãao ${ }^{1,12}$.

Todas as lesões eram notificadas ao departamento médico, que listava a posição de jogo do atleta, a circunstância (com ou sem contato), região anatômica, a gravidade da lesão e o tempo (dias) de afastamento e tratamento, baseados no Orchard Sports Injury Classification System ${ }^{13,14}$.

A classificação de gravidade da lesáo foi baseada no período de afastamento das atividades esportivas, dessa forma: lesão leve, tempo $\leq 1$ semana de afastamento; lesão moderada: tempo > 1 semana $<1$ mês de afastamento; e lesão grave, $>1$ mês de afastamento ${ }^{15}$.

\section{Análise estatística}

As análises foram realizadas no Statistica 12. A análise descritiva foi realizada por meio da média e do desvio-padrão para os dados contínuos de frequência e porcentagem para os categóricos.

A quantidade de lesóes foi expressa pela ocorrência em jogos ou treinos, ou por lesóes a cada 1000 horas de jogo ou treino ${ }^{16}$.

O tempo de exposição ao risco foi calculado multiplicando a quantidade de atletas pelas horas praticadas. Para a exposiçáo ao tempo de jogo, foi multiplicada a quantidade de horas de jogos pela quantidade de atletas que entram em quadra, e para a exposição ao treino, foi multiplicada a quantidade de horas de treino pelo total de atletas ${ }^{16}$.

\section{Resultados}

Vinte voluntários com idade média de 17,5 $\pm 2,5$ anos iniciaram o estudo, entretanto, foram excluídos quatro atletas, por doenças sistêmicas no decorrer da temporada. Os atletas competiram 42 horas e treinaram 106 horas, com tempo de exposição de 294 horas de jogo e de 1.696 horas de treino, com total de 1.990 horas de exposição.

Dos 16 voluntários estudados, $13(81,25 \%)$ se lesionaram durante a temporada, totalizando 26 lesóes que resultaram em afastamento. Dessas, 14 (53,84\%) foram lesōes recorrentes.

A quantidade de lesôes foi maior durante o treino $(54,85 \%)$ do que no jogo $(46,15 \%)$. A incidência total foi de 13,06 lesóes por 1.000 horas praticadas, sendo que durante os jogos foi de 40,81 lesôes/1000 horas e nos treinos de 8,24 lesóes/1000 horas (Tabela 1).

O tempo de afastamento foi de $8 \pm 10,9$ dias (lesões novas: $6 \pm 11,54$ dias; recorrentes: $9 \pm 10,32$ dias). A região anatômica mais acometida foi o tornozelo (41,66\%), seguido pelo ombro (25\%), joelho (12,5\%), cabeça $(8,33 \%)$, mão $(4,16 \%)$, coxa $(4,16 \%)$ e cotovelo $(4,16 \%)$ (Tabela 2). 
Tabela 1: Características das lesões.

\begin{tabular}{lc}
\hline Variáveis & Quantidade \\
\hline Lesóes & 26 \\
Lesóes durante os jogos / 1000 horas & 40,81 \\
Lesóes durante os treinos / 1000 horas & 8,24 \\
Circunstância* & $7(27)$ \\
Com contato & $19(73)$ \\
Sem contato & \\
Tempo de Afastamento* & $11(42,3)$ \\
1 dia & $3(11,5)$ \\
$2-7$ dias & $12(46)$ \\
1 semana - 1 mês & \\
\hline
\end{tabular}

*Valores expressos em frequência (\%).

Tabela 2: Distribuição das lesões, dias de afastamento e tratamento.

\begin{tabular}{|c|c|c|c|}
\hline Lesão & Quantidade & Afastamento* & Tratamento* \\
\hline \multicolumn{4}{|l|}{ Membro inferior } \\
\hline Contusáo/hematoma do tornozelo & 2 & $11 \pm 14,1$ & $41 \pm 6,3$ \\
\hline Entorse lateral de tornozelo & 9 & $12 \pm 11,6$ & $25 \pm 9,2$ \\
\hline Tendinopatia de Aquiles/ Bursite retrocalcânea & 1 & 3 & 30 \\
\hline Estiramento dos músculos adutores & 1 & 14 & 21 \\
\hline Lesáo condral do compartimento patelofemoral & 1 & 2 & 112 \\
\hline Contusão/hematoma do joelho & 1 & 2 & 14 \\
\hline Tendinopatia patelar & 1 & 7 & 30 \\
\hline \multicolumn{4}{|l|}{ Membro superior } \\
\hline Luxaçáo da primeira articulaçáo metacarpofalangeana & 1 & 2 & 60 \\
\hline Estiramento muscular/regiáo do ombro & 3 & $7 \pm 7,5$ & $11 \pm 47,9$ \\
\hline Epicondilite medial & 1 & 7 & 60 \\
\hline Acometimento crônico subacromial & 3 & $7 \pm 8,5$ & $250 \pm 36$ \\
\hline \multicolumn{4}{|l|}{ Cabeça e face } \\
\hline Laceração do queixo & 1 & 2 & 0 \\
\hline Fratura de dente & 1 & 21 & 0 \\
\hline Total & 26 & $10,7 \pm 10,5$ & $52,3 \pm 68,1$ \\
\hline
\end{tabular}

*Valores expressos por média $\pm \mathrm{DP}$.

Dentre as lesões, $75 \%$ foram leves, $25 \%$ foram moderadas, e não houve a ocorrência de lesóes graves que resultassem na restrição de mais de um mês sem a prática esportiva (Tabela 1 ).

O mecanismo de lesão mais comum foi o uso excessivo $(37,5 \%)$, seguido pelo trauma $(29,16 \%)$, entorse
$(29,16 \%)$ e estiramento $(4,16 \%)$ (Tabela 3). Dentre os atletas estudados, $23,07 \%$ jogavam na posição de pivô, $23,07 \%$ de meia, $23,7 \%$ de ponta, $15,38 \%$ de central e $15,38 \%$ de goleiro. As lesóes que ocorreram durante competição e treino, e o tempo de afastamento estão na Tabela 3.

Tabela 3: Lesões nas competições e treinos e dias de afastamento.

\begin{tabular}{lcccc}
\hline \multirow{2}{*}{ Lesáo } & \multicolumn{2}{c}{ Competiçáo } & \multicolumn{2}{c}{ Treino } \\
\cline { 2 - 5 } & Quantidade & Afastamento* & Quantidade & Afastamento* \\
\hline Fratura e estresse ósseo & 1 & 21 & - & - \\
Articulaçáo e ligamento & 8 & $17 \pm 13,3$ & 3 & $7 \pm 6,5$ \\
Músculo e tendáo & 2 & $10 \pm 14,1$ & 10 & $7 \pm 6,8$ \\
Laceraçáo e lesáo de pele & 1 & 1 & 1 & 1 \\
Total & 12 & $15,4 \pm 25,3$ & 13 & $5 \pm 6,5$ \\
\hline
\end{tabular}

*Valores expressos por média \pm DP. 


\section{Discussão}

A maior ocorrência de lesóes durante os treinos pode ter ocorrido pela maior quantidade de horas treinadas do que competidas. Entretanto, quando observada a incidência de lesóes por 1000 horas praticadas, a quantidade de lesóes ocorridas durante os jogos foi maior. Em outros estudos ${ }^{8,10}$, houve mais lesóes durante os jogos, e podem ter ocorrido pela maior intensidade, disputa e velocidade do jogo em relação ao treino.

A maioria dos estudos documentam apenas as lesóes que causam afastamento de um treino ou jogo, porém se forem consideradas as lesôes menos graves, que não afastam os atletas de suas atividades esportivas, a incidência pode chegar a 108 lesóes/1.000 horas competidas ${ }^{1}$, sendo que essas lesões são de importância epidemiológica, pois influenciam o desempenho físico, e pode ser um fator desencadeante de outras lesōes.

As regiōes mais acometidas foram o tornozelo e o ombro, concordando parcialmente com os achados de Yde e Nielsen ${ }^{17}$, que encontraram em atletas de handebol um maior acometimento do tornozelo (40\%), seguido pela máo e dedo $(25 \%)$ e joelho $(14 \%)$. O mesmo foi observado por Dirx et al. ${ }^{18}$, que relataram um acometimento predominante do tornozelo (35\%), seguido pelo dedo (16\%) e joelho (12\%). Outros autores ${ }^{11,19,20}$ também encontraram maior acometimento dos membros inferiores, seguido pelos superiores e tronco.

Estratégias de prevenção podem auxiliar a redução dessas lesôes. Alguns estudos observaram que intervençóes podem ser utilizadas na prevenção de lesôes nos membros inferiores, como exercícios excêntricos, pliométricos e sensório-motor, fortalecimento dos músculos abdutores e rotadores laterais de quadril, utilização de órteses para o tornozelo ${ }^{21,22}$. Outras pesquisas observaram alteraçóes no ombro de arremessadores, como diminuição da rotação medial de ombro e discinesia escapular, que podem originar lesóes ${ }^{23,24}$.

Algumas limitaçóes deste estudo devem ser consideradas. Não foram considerados alguns fatores que podem influenciar o trabalho epidemiológico envolvendo lesôes no handebol, como a utilização de bandagens e outros equipamentos de proteção $0^{4}$, condiçóes climáticas, tipo de superfície do chão e calçados $^{25-27}$.

Assim, são necessários estudos longitudinais abrangendo maior quantidade de atletas por período prolongado de tempo, além de avaliar a influência de fatores que podem estar associados às lesôes, formando bases específicas e adequadas para a prevenção e tratamento.

\section{Conclusão}

As lesóes observadas acometeram predominantemente os membros inferiores seguidos pelos superiores, sendo a maioria considerada leve. A quantidade de lesões durante os treinos foi maior, mas quando considerada por hora praticada, os jogos foram mais lesivos. Pesquisas futuras são necessárias para avaliar a influência de programas preventivos sobre essas lesóes.

\section{Referências}

1. Langevoort G, Myklebust G, Dvorak J, Junge A. Handball injuries during major international tournaments. Scand J Med Sci Sports. 2007;17(4):400-7.

2. Ghobadi H, Rajabi H, Farzad B, Bayati M, Jeffreys I. Anthropometry of World-Class Elite Handball Players According to the Playing Position: Reports From Men's Handball World Championship 2013. J Hum Kinet. 2013;39:213-20.

3. Abernethy L, Bleakley C. Strategies to prevent injury in adolescent sport: a systematic review. Br J Sports Med. 2007;41(10):627-38.

4. Vlak T, Pivalica D. Handball: the beauty or the beast. Croat Med J. 2004;45(5):526-30.

5. Junge A, Engebretsen L, Mountjoy ML, Alonso JM, Renstrom PA, Aubry MJ, et al. Sports injuries during the Summer Olympic Games 2008. Am J Sports Med. 2009;37(11):2165-72.

6. Chesnin KJ, Selby-Silverstein L, Besser MP. Comparison of an in-shoe pressure measurement device to a force plate: concurrent validity of center of pressure measurements. Gait Posture. 2000;12(2):128-33.

7. Seil R, Rupp S, Tempelhof S, Kohn D. [Injuries during handball. A comparative, retrospective study between regional and upper league teams]. Sportverletz Sportschaden. 1997;11(2):58-62.

8. Reckling C, Zantop T, Petersen W. [Epidemiology of injuries in juvenile handball players]. Sportverletz Sportschaden. 2003;17(3):112-7.

9. Oehlert K, Drescher W, Petersen W, Zantop T, Gross $\mathrm{V}$, Hassenpflug J. [Injuries in Olympic handball tournaments: a video analysis]. Sportverletz Sportschaden. 2004;18(2):80-4.

10. Olsen OE, Myklebust G, Engebretsen L, Bahr R. Injury pattern in youth team handball: a comparison of two prospective registration methods. Scand J Med Sci Sports. 2006;16(6):426-32.

11. Nielsen AB, Yde J. An epidemiologic and traumatologic study of injuries in handball. International journal of sports medicine. 1988;9(5):341-4.

12. Huffman EA, Yard EE, Fields SK, Collins CL, Comstock RD. Epidemiology of rare injuries and conditions among 
United States high school athletes during the 2005-2006 and 2006-2007 school years. J Athl Train. 2008;43(6):624-30.

13. Rae K, Orchard J. The Orchard Sports Injury Classification System (OSICS) version 10. Clin J Sport Med. 2007;17(3):201-4.

14. Orchard J, Rae K, Brooks J, Hagglund M, Til L, Wales $\mathrm{D}$, et al. Revision, uptake and coding issues related to the open access Orchard Sports Injury Classification System (OSICS) versions 8, 9 and 10.1. Open Access J Sports Med. 2010;1:207-14.

15. Silva ASd, Abdalla RJ, Fisberg M. Incidência de lesôes musculoesqueléticas em atletas de elite do basquetebol feminino. Acta Ortop Bras. 2007;15:43-6.

16. Phillips LH. Sports injury incidence. Br J Sports Med. 2000;34(2):133-6.

17. Yde J, Nielsen AB. Sports injuries in adolescents' ball games: soccer, handball and basketball. Br J Sports Med. 1990;24(1):51-4.

18. Dirx M, Bouter LM, de Geus GH. Aetiology of handball injuries: a case-control study. $\mathrm{Br} J$ Sports Med. 1992;26(3):121-4.

19. Habelt S, Hasler CC, Steinbruck K, Majewski M. Sport injuries in adolescents. Orthop Rev. 2011;3(2):7.

20. Chen S-K, Cheng Y-M, Huang P-J, Chou P-H, Lin Y-C, Hong Y-J. Investigation of Management Models in Elite Athlete Injuries. The Kaohsiung J Med Sci. 2005;21(5):220-7.
21. Emery C, Meeuwisse W. The effectiveness of a neuromuscular prevention strategy to reduce injuries in youth soccer: a cluster-randomised controlled trial. Br J Sports Med. 2013;44(4):555-62.

22. Chimera N, Swanik K, Swanik C, Straub S. Effects of Plyometric Training on Muscle-Activation Strategies and Performance in Female Athletes. J Athl Train. 2004;39(1):24-31.

23. Almeida GPL, Silveira PF, Rosseto NP, Barbosa G, Ejnisman $\mathrm{B}$, Cohen M. Glenohumeral range of motion in handball players with and without throwing-related shoulder pain. J Shoulder Elbow Surg. 2013;22(5):602-7.

24. Forthomme B, Crielaard J, Croisier J. Scapular Positioning in Athlete's Shoulder Particularities, Clinical Measurements and Implications. Sports Med. 2008;38(5):369-86.

25. Olsen OE, Myklebust G, Engebretsen L, Holme I, Bahr R. Relationship between floor type and risk of ACL injury in team handball. Scand J Med Sci Sports. 2003;13(5):299-304.

26. Di Caprio F, Buda R, Mosca M, Calabro A, Giannini S. Foot and lower limb diseases in runners: assessment of risk factors. J Sports Sci Med. 2010;9(4):587-96.

27. Wilk B, Fisher K, Gutierrez W. Defective running shoes as a contributing factor in plantar fasciitis in a triathlete. J Orthop Sports Phys Ther. 2000;30(1):21-8.

\section{Como citar este artigo:}

Kamonsekia DH, Fonseca CL, Zamunér AR, Souza NA, Aguiar GG, Peixoto BO. Incidência de lesóes musculoesqueléticas em atletas masculinos de handebol. Rev. Aten. Saúde. 2015;13(46):31-5 